\title{
The Median Survival Recovery Time and Associated Factors Among Admitted Neonate in Intensive Care Units of Dire Dawa Public Hospitals, East Ethiopia, 2019.
}

\author{
Tariku Derese ( $\nabla$ t.derese@yahoo.co.uk) \\ Dire Dawa University \\ Yalelet Belay \\ Dire Dawa University \\ Zerihun Tariku \\ Dire Dawa University
}

Research article

Keywords: Neonates, survival, Recovery, Time, Dire Dawa

Posted Date: April 28th, 2020

DOI: https://doi.org/10.21203/rs.3.rs-22899/v1

License: (c) (i) This work is licensed under a Creative Commons Attribution 4.0 International License.

Read Full License 


\section{Abstract \\ Background}

Neonate is any infants from the birth to 28 days. Most of the neonatal deaths occur in developing countries particularly in sub-Saharan African and south central Asian countries. The ability to predict Length of stay would be valuable to parents and families, clinicians and service providers. Timely Management and treatment of birth complication are important factors in reducing new born mortality.

\section{Objective}

The median Survival Recovery time and associated factors among admitted neonate in intensive care units of Dire Dawa Governmental Hospitals, East Ethiopia, 2019.

\section{Methods}

Facility based retrospective cohort study design was employed to assess recovery time and associated factors of neonate among a total of 499 selected 0-28 days of neonates from two public Hospitals and validated Checklist were used to assess data. Data were entered in to Epi-data version 3.1 and exported to SPSS window version 21.0. Descriptive data were presented by table and graph. To determine the associated factors, Cox regression model was computed with $95 \% \mathrm{Cl}$ and P-value $<0.05$ level of significance. Ethical clearance was taken from Dire Dawa University research and technology interchange office and given for all concerned body.

\section{Result}

The overall median survival recovery time of neonates admitted in neonatal intensive care units of Dire Dawa public hospital was 7 Days with $95 \% \mathrm{Cl}(6.525-7.475)$. Among the neonates that admitted in neonatal intensive care units, neonates those who have weight $<2500 \mathrm{~g}$ had 1.648 times higher hazard risks to recovery compared to that neonates who have weight greater than or equal to $4000 \mathrm{~g}$ with [AHR $1.64895 \% \mathrm{Cl}(1.246-2.179)]$. Those neonates who were none intubated had 6.725 higher hazard risks compared to that of intubates neonates [AHR 6.725 95\% Cl (1.616-27.978)], and those neonates who were not supply oxygen continuously had 1.336 times low probability to for recovery [AHR $1.33695 \% \mathrm{Cl}$ (1.030-1.733)]. But, the neonates admitted between 1-6 days after birth had 0.521 times higher probability for recovery compared to the neonate with those neonate admitted between 7-28 days of after birth [AHR $0.52195 \% \mathrm{Cl}(0.355-0.763)]$.

\section{Conclusion}


Overall median survival recovery time was 7 Days and Birth weight of neonates, Oxygen supply, and Intubation and admission time of neonate between 1-6 days of after birth were factors that significantly associated with recovery time of neonates. Compared to the other study the recovery time of neonates in this study were short and better.

\section{Background}

A neonate is any infant aged less than 28 completed days. Neonatal units may provide care for infants up to 6 months postnatal or two months of age corrected for prematurity. Newborn All babies in hospitals are admitted patients and are defined as either qualified or unqualified (1). Worldwide there are 6.3 million Neonatal deaths a year, almost all occurs in developing countries, and $27 \%$ of them in the least developed countries alone (2). More than $40 \%$ of all under five Deaths occur during the neonatal period at the first month of life (3).

Among 140 million babies born worldwide, 90\% were born in low developing countries and 10\% in high developed countries while approximately $99 \%$ neonatal death occurs in low income countries. Every minute seven new born babies die world wide and 415 new born babies every hour. The vast majority of neonatal death occurs in South Asian and Sub-Sahara Africa (4).

Most of the neonatal deaths occur in developing countries particularly in sub-Saharan African and south central Asian countries. Despite improvements over the past decade, Ethiopia's current neonatal mortality rate is one of the highest in the world. Neonatal mortality comprises a significant proportion of the underfive mortality in the country (5).

SDG had sets a goals by the end of 2030, end preventable newborns and child under 5 years of age, with all countries aiming to reduce neonatal mortality to at least as low as 12 per 1000 live births and under 5 mortality to at least as low as 25 per 1000 live births(6)

But, EDHS 2016 report showed that, in Ethiopia the under-5 mortality rate is 67 deaths per 1,000 live births, and the infant mortality rate is 48 deaths per 1,000 live births. This means that 1 in 15 children in Ethiopia dies before reaching age 5 , and 7 in 10 of the deaths occur during infancy. Results showed that the neonatal, infant, and under- 5 mortality rates for the 5 years before the survey are 29, 48, and 67 deaths per 1,000 live births, respectively. In other words, in Ethiopia 1 in every 35 children dies within the first month, 1 in every 21 children dies before celebrating the first birthday, and 1 of every 15 children dies before reaching the fifth birthday. However, the change in neonatal mortality is not as significant as the change in post-neonatal and child mortality. Even in Dire Dawa the neonatal mortality rate remain $36 / 1000$ live birth which is higher compared to Harari and Tigray regions and 2time higher than Addis Ababa (7).

Preterm newborns had hospital stays averaging 14.3 days. Among newborns with low birth weight, those who weighed less than 1500 grams had hospital stays 42.6 days compared to 10.9 days 1500 grams or 
more. Newborns with respiratory distress syndrome had hospital stays averaging 31.3 days. Newborns that died after birth had hospital stays averaging 7.4 days (8)

The ability to predict Length of stay would be valuable to parents and families, clinicians and service providers, but it is a complex issue. Inherent factors appear to be the most important and this information from the first day of life is informative for predicting Length of stay in a simple model and these estimates are a useful early indicator of Length of stay (9)

Timely Management and treatment of birth complication are important factors in reducing new born mortality. To give new born access to life saving care, standard guidelines recommend that all births take place in a health facility under the care of skill health provider (10).

The ability that accurately predicts length of stay in Neonatal care is vital in resource planning, commissioning services and to aid clinician in their counseling of parents. It is vital information about the factors which predict length of stay is identify from multiple studies for the future research (11).

Therefore this study aims to assess median survival recovery time and associated factors that lead to compromise the recovery of neonate in the intensive care unit.

\section{Methods}

\section{Study area and period}

The study was conducted at DilChora Referral and Sabian primary Hospitals, in Dire Dawa, which is located 515 kilometers away East of Addis Ababa.

A Dilchora referral hospital had 24 functional NICU Beds, 2 functional Incubators and 18 total staff working the neonatal intensive care units. Among the total of 18 staff, One Neonatal Bsc Nurse, 8 Intensive neonatal cares trained and 2 untrained nurses, 1 General Practitioner, 2 runners and 4 cleaners were working a full time in the unit. Sabian Primary had 15 Neonatal Beds, 3 functional Incubators, 1 neonatal nurse and 3 trained nurses.

Dilchora Referral hospital neonatal intensive care unit established ten years ago, but Sabian primary hospital neonatal intensive care unit established in 2014/15. Data was collected from May 20/05/2019June 15/06/2019

\section{Study Design}

Facility Based retrospective cohort study design was implemented and standardized checklist was used to assess the neonatal recovery time and risk factors associated with neonatal recovery.

\section{Inclusion and Exclusion Criteria}

\section{Inclusion criteria}


Neonates admitted in Dilchora referral and Sabian primary Hospital's Intensive Neonatal care units in any case from January 10/01/2017 to January 10/01/2019.

\section{Exclusion Criteria}

Neonates with incomplete charts January 10/01/2017 to January 10/01/2019.

Neonates admitted for less than $24 \mathrm{hrs}$ in neonatal intensive care units.

\section{Operational definitions}

'Recovery': were neonates that declared as improved or recovered by physician.

Median Survival Recovery time: is the median survival recovery of time of neonates that declared recovered from illness by physician working in the units.

Admission: neonate admitted by physicians and wait for more than $24 \mathrm{hrs}$

'Defaulters'- were those neonates who leaves from treatment unit against medical advice and declared as defaulter or against in the treatment.

'Death'- Refers to the neonates that has die while he/she is in the therapeutic units and declared as Death in the treatment logbook by physician.

Recovery rate= Number of neonates discharged for recovery or improve $\times 100$

Total admission cases with in the study period

Defaulter rate $=\underline{\text { Number of defaulters/against from the treatment unit } \times 100}$

Total admission cases with in the study period

Death rate $=$ Total number of death in the therapeutic units $\times 100$

Total admission cases with in the study period

Censor=Refers to Defaulter from treatment, transfer out, referred neonates, those who died with indirect and direct causes and those cases not known the result at the end of the study period.

The event (outcome of interest) =was considered survival median recovery time of neonate while in the neonatal intensive care units.

\section{Data Quality control}

The data was collected by using standardized checklist and reviewing a chart which was adopted from previous Literature $(12,13)$ and Data collection checklist was also, developed from the existing register of 
the Hospitals to address the study variables. The data collection checklist was checked for consistency and during data collection the discrepancies of the data was manage by third person. Data clearance, Completeness, range and logic checks carry out regularly to ensure the quality of data before data entry. Any data that were incomplete and inconsistent was excluding from the data and checks with the supervisors.

\section{Data collection procedure}

Data source was neonatal care unit registration logbook and charts of each case from the units Data was collected by Nurses who took training on the management of NICU from the other hospitals and they were collect data by standardized checklist. Six data collectors and two supervisors were trained for two day about how and what information was collected. The investigator was monitoring the data collectors to ensure the quality of data that was collected.

\section{Data processing and Analysis}

The data were entered into Epi data version 3.1 and analysis was done by using SPSS Version 21 software. Variable associated with time to recovery at 0.2 significant levels was included in the multivariate Cox proportional Hazard model. The associated factors was compared by hazard function risks and data was presented by Log survival function Graphs. Variables having $p<0.05$ was considered as significant.

\section{Results}

\section{Socio-Demographic characteristics of recovery time of neonate admitted in Dire Dawa} Governmental Hospitals, 2019.

Among the neonates aged 0-28 days, majority 66.5( $\mathrm{n}=499)$ were age group from zero to 1 days with the mean age of 1.44 days +_0.675 SD. There were $64.7 \%$ (323) male and $35.3 \%$ (176) female admitted to neonatal intensive care units in both hospitals. The majority $(55.1 \% \mathrm{n}=499)$ of admitted neonate in intensive care units were from rural area and the rest $44.9 \%$ were from the urban (table1).

Place of Delivery, referral made and type of neonatal sepsis, in Dire Dawa Administration governmental hospitals

Admission criteria and Management in neonatal care units were accordance with national guideline for treatment of neonates. Among admitted neonates in Neonatal care units 66.1\% (330) were delivered in hospitals and 9\% (45) were delivered at Home (Figure 1). The majority $61.1 \%$ (305) referred to neonatal intensive care units from the same Facility, $22 \%$ (110) from Health Centre, 8.8 (44) from other hospitals and $8 \%(40)$ were referred from home. The predominant form of sepsis $87.6 \%(n=315)$ was early onset of sepsis and $12.4 \%$ was late onset of neonatal sepsis.

Admission Case and treatment Outcome of neonates admitted in Dire Dawa Governmental hospitals in Intensive care Units. 
The main neonatal treatment outcome indicators were recovery rate, Death rate, Defaulter and transfer out rate. Among 499 admitted neonates in neonatal intensive care units, $84.4 \%, 8.8 \%, 3 \%$ and $3 \%$ were recovered, died, Defaulted and transferred respectively. Most of the neonates were recovered $421(84.4 \%)$ and $44(8.8 \%)$ were reported as died. The predominant cases of admission were early onset of neonatal sepsis $168(33.7 \%)$. Prematurity $10(22.7 \%)$ and the complicated cases $10(22.7 \%)$ had higher deaths rate compared to the others (table 2).

Mode of delivery and admission time of neonates after birth in neonatal intensive care units in Dire Dawa Governmental hospitals from January 2017 to January 2019.

The predominant $77 \%(n=499)$ neonates were vaginal delivery and other $20.8 \%, 2.2 \%$ were $C$-section and Instrumental assisted delivery respectively. The majority $278(55.7 \%), n=499)$ of the neonates were admitted with in $24 \mathrm{Hrs}$. Most of the neonatal death 33 (75\%) occurs in the first $24 \mathrm{hrs}$ of birth (Figure 2).

The median survival recovery time of neonates admitted in Dilchora referral hospital was 6 days with $95 \% \mathrm{Cl}(5.472-6.528)$ and the median survival recovery time of neonates admitted in neonatal intensive care unit of Sabian primary Hospital was 8 days with $95 \% \mathrm{Cl}$ (6.99-9.001). The overall median survival recovery time of neonates admitted in neonatal intensive care units of Dire Dawa Governmental hospital was 7 Days with $95 \% \mathrm{Cl}(6.525-7.475)$ (Table 3$)$.

\section{Log survival function Estimate of recovery time and admission time after birth}

The log rank test showed that admission time after birth and recovery time has a significant association with $p$-value (0.019). The log survival function graph showed that, there is a significant difference for survival of neonates based on admission time after birth and with length of stay. Mortality and censoring was higher with in $24 \mathrm{rs}$. The log survival function graph indicates, $75 \%$ death occurred in the first 24 hours of birth and Neonates admitted between 7-28 days had a lower probability for death (Figure 3).

\section{Factors associated with median survival recovery time of neonates admitted to in neonatal Intensive care units.}

The Final Backward conditional steps of cox regression analysis showed that, Birth weight of neonates, Presence of hypothermia, low birth weight, provision of antibiotic, and Hypothermia, Intubation and admission time of neonate after birth were factors that significantly associated with recovery time of neonates admitted in the neonatal intensive care units. Among the neonates that admitted in neonatal intensive care units, neonates those who have weight $<2500 \mathrm{~g}$ had 1.648 times higher hazard risks to recovery compared to that neonates who have weight greater than or equal to $4000 \mathrm{~g}$ with [AHR 1.648 $95 \% \mathrm{Cl}(1.246-2.179)]$. Those neonates who were none intubated had 6.725 higher hazard risks compared to that of intubates neonates [AHR 6.725 95\% Cl (1.616-27.978)], and those neonates who were not supply oxygen continuously had 1.336 times low probability to for recovery [AHR $1.33695 \% \mathrm{Cl}(1.030$ 1.733)]. But, the neonates admitted between 1-6 days after birth had 0.521 times higher probability for recovery compared to the neonate with those neonate admitted between 7-28 days of after birth [AHR $0.52195 \% \mathrm{Cl}(0.355-0.763)$ ]. Birth weight 2500g-3999g [AHR 3.047 95\% Cl (1.439-6.451)] were also significantly associated with recovery time (table 4). 


\section{Discussion}

This study showed that, most of neonates admitted in neonatal intensive care units were from rural area $55.1 \%(n=499)$ and there were $64.7 \%$ (323) male and 35.3\% (176) female. The study conducted in university of Gonder Specialized hospital showed similar finding, there were 448 (58.3\%) male neonates. Regarding the residency, 398 (51.8\%) neonates were from rural area (12).

Most of admitted neonates in Neonatal care units 66.1\% (330) were delivered in hospitals and $9 \%(45)$ were delivered at Home. The predominant form of sepsis $87.6 \%$ (276) was early onset of sepsis and the other $12.4 \%$ (39) were late onset of sepsis. The study conducted in University of Gonder showed that, more than two-thirds of the 587 deliveries (76.3\%) were performed in hospital. On the other hand the finding from Shashemene governmental hospital among 244 neonates who were admitted in NICU, $190(77.9 \%)$ had neonatal sepsis. From this were 123 (64.7\%) who had early onset neonatal sepsis and 67 $(35.3 \%)$ who had late onset neonatal sepsis $(12,13)$. The result shows a difference, this may be due to time gaps and sepsis prevalence is highly depends on the IPPS Implementation practice of each hospitals.

Out of 499 admitted neonates, in neonatal intensive care units $84.4 \%, 8.8 \%, 3 \%$ and $3 \%$ were recovered, died, Defaulted and transferred respectively. Predominant cases of admission were early onset of neonatal sepsis (33.7\%), more than one case (20.2\%), prematurity $(9.4 \%)$ and respiratory distress $(9 \%)$. The finding from Addis Ababa showed that, the most common primary diagnoses during admission to the neonatal care unit were prematurity with respiratory problem (36.6\%), neonatal sepsis $(22.7 \%)$, and asphyxia (16.2\%) which was different with this study. But, the study from Ghana Tamale teaching hospital showed that, the commonest cause of neonatal admission was sepsis (29.2\%), followed by prematurity/low birth weight $(26.9 \%)$, birth asphyxia (16.2\%) and congenital anomalies (7.1\%). Majority $82.7 \%(3220)$ of the neonates were successfully treated and discharged $(14,15)$. The treatment success of this study were better compared to Tamale teaching hospital. This may be due to the difference of the strategic focus of these two countries and available human skill as well as Geographical variation.

The majority $278(55.7 \%), n=499)$ of the neonates were admitted with in $24 \mathrm{Hrs}$. Most of the neonatal death $33(75 \%)$ occurs in the first $24 \mathrm{hrs}$ of birth and as the length of stay increase the probability of neonate recovery also increase. The study find from university of Gonder indicate, The overall mortality was 110 (14.3\%; of which $69(62.7 \%)$ deaths occurred in the first 24 hours of age(12) which indicate high number of early mortality rate, but show a difference in magnitude. This may be due to sample size and study time difference.

The overall median survival recovery time of neonates admitted in neonatal intensive care units of Dire Dawa Governmental hospital was 7 Days with $95 \% \mathrm{Cl} 0.243(6.525-7.475)$. The study finding from Brazil indicate that the mean newborns stay length among those who were discharged was 19 days, while the mean stay length for those who died was 8 days (16), Which show very different result compared to this study. The variation may be due to Geographical difference and admission case difference. 
The Final Backward conditional steps of cox regression analysis showed that, Birth weight of neonates, oxygen supply, Intubation and admission time of neonate 1-6 days of after birth were factors that significantly associated with recovery time.

Univar ate Analysis from Iran Conducted to evaluate the risk factors associated with complications revealed that gestational age of $<37$ weeks, mean birth weight, and Intubation before transfer had a significant association with transfer complication(17). On the other hand, study finding in Nigeria showed that, neonatal admission in the first 24 hours of birth, Neonates with low birth weight $(p<0.05)$ and neonates birth weight were significantly associated with neonatal mortality (18)

\section{Conclusion}

The most commonly admitted age groups of neonates were between 0-24 Hours old age. The predominant case of admission in neonatal intensive care units was Neonatal sepsis. The majority of neonates admitted in intensive care units were delivered in hospitals. The recovery rate of this study was $84.4 \%$. Hazard function graph indicates, $75 \%$ death occurred in the first 24 hours of birth and Neonates admitted between 7-28 days had a lower probability for death.

Finally, overall median survival recovery time of neonates admitted in neonatal intensive care units of Dire Dawa public hospitals was 7 Days. The Final Backward conditional steps of cox regression analysis this study showed that, Birth weight, Intubation, Oxygen supply and admission between 1-6 days after birth were significantly associated with recovery time.

The finding showed that the recovery time of neonates in this study is better than the other studies conducted in different part of sub-sahara countries.

\section{Abbreviations}




\begin{tabular}{ll} 
AHR & Adjusted Hazard Ratio \\
\hline Cl & Confidence Interval \\
\hline CMHS & College of medicine and health science \\
\hline DDHB & Dire Dawa Health Bureau \\
\hline DRH & Dilchora referral hospital \\
\hline EDHS & Ethiopia Demography Health survey \\
\hline FMOH & Federal Ministry of Health \\
\hline HR & Hazard Ratio \\
\hline IPPS & Infection prevention and patient safety \\
\hline MNCH & Maternal, neonate and child health \\
\hline OR & Odd Ratio \\
\hline NAS & Nursing Assessment and study \\
\hline NICU & Neonatal intensive care unit \\
\hline RTI & Research and technology interchange \\
\hline SDG & Sustainable Development Goal \\
\hline SPH & Sabian primary hospital \\
\hline UNICEF & United nation child fund \\
\hline WHO & World Health Organization \\
\hline
\end{tabular}

\section{Declaration}

\section{Ethics Approval and Consent to participate}

Official support letter was obtained from Dire Dawa University Research and technology Inter-change office and the letter was given to Dire Dawa town administration Health Bureau, then for Dilchora and Sabian Hospitals chief executive officer, finally for unit coordinators.

During data collection the purpose of the study was explained for the data owners. The permission of Hospitals medical room coordinators was obtained before chart review and street confidentiality was kept by reviewers. No information disclose to any third person that obtain from the medical records. Each medical chart was review and returned carefully by checking the list of each medical record numbers with proper care. 
Consent for publication is not necessary, because this manuscript did not contain any personal detail like photo, image and video data.

\section{Availability of data and Material}

The data sets used or analyzed data currently availed in the hand of corresponding author and can be asked any time for reasonable request.

\section{Competing interests}

Authors declared that, there is no competing interest regarding to this manuscript.

\section{Funding}

Dire Dawa University is the full funder of this research article. Dire Dawa University was responsible for validation of the Quality of the paper as well as follows the whole process of the paper based on scientific steps and procedures.

\section{Author's contribution}

TD develops, organized the materials and designs the study, perform analysis and interpret the data. ZT assists the methods and YB evaluates the contents. TD also writes the manuscript and all the authors read and approved the final manuscript

\section{Acknowledgments}

First of all, we would like to thanks Dire Dawa University for giving us this grant for completing this research paper from which we learnt a great lesson.

This is also a right time to us to express our great thanks to Ms. Aliya Nuri (CMHS, research and community engagement coordinator) and Dire Dawa university research and technology interchange office for their invaluable guidance and support throughout this research paper work.

Finally, it is our pleasure to thanks Dire Dawa University Finance workers Mr Belete and Ms Azeb for sharing their knowledge and skill of financial management throughout the process.

\section{References}

1. Department of Health, Western Australia. Framework for the care of neonates in Western Australia. Perth: Health Networks Branch, Department of Health, Western Australia; 2009.

2. World health organization. Neonatal and perinatal mortality, country, regional and global estimates:2006

3. Jayani P Flor M and Vectoria A. neonatal death: Case definition, guideline for data collection, analysis and presentation of immunization safety data. Vol 34, isseu49, 2016 
4. Sajjad UR Lahman and Walid El Alsari. Neonatal mortality: Incidence, correlates and improvement strategies, Perinatal mortality. 2012

5. Mekonnen Y, Tensou B, Telake DS, Degefe T, Bekele A. Neonatal mortality in Ethiopia: trends and determinants. BMC Public Health.2013;13:483

6. UN: The 2030 agenda for sustainable Development Goals.

7. Central Statistical Agency (CSA) [Ethiopia] and ICF. 2016. Ethiopia Demographic and Health Survey 2016. Addis Ababa, Ethiopia, and Rockville, Maryland, USA: CSA and ICF.

8. Kowlessar NM (Social \& Scientific Systems, Inc.), Jiang HJ (AHRQ), and Steiner C (AHRQ).Hospital Stays for Newborns, 2011. HCUP Statistical Brief \#163. October 2013.Agency for Healthcare Research and Quality, Rockville, MD. http://www.hcup-us.ahrq.gov/reports/statbriefs/sb163.pdf.

9. Seaton SE, Barker $L$, Jenkins $D$, et al. What factors predict length of stay in a neonatal unit: a systematic review. BMJ Open 2016;6:e010466. doi:10.1136/bmjopen-2015-010466..

10. UNICEF and Safe the children. New born health in humanitarian settings: 2015

11. http://pediatrics.applications.org.2017

12. Abaynesh G, Fantahun A and Mahlet A. Pattern of admission and factors associated with neonatal mortality among the neonate admitted to the neonatal intensive care unit of University of Gondar Hospital, North West Ethiopia . pediatrics health, medicine and therapuitics.2017

13. Aytenew Getabelew et al. Prevalence of Neonatal Sepsis and Associated Factors among Neonates in Neonatal Intensive Care Unit at Selected Governmental Hospitals in Shashemene Town, Oromia Regional State, Ethiopia, 2017.

14. Atnafu et al : Reasons for admission and neonatal outcome in the neonatal care unit of a tertiary care hospital in addis ababa: a prospective study: Research and Reports in Neonatology 2016:6 1723

15. Walana W, Acquah Ekuban KS, Abdul-Mumin A, Naafu B, Aruk E, et al. (2016) Pattern, Causes and Treatment Outcomes of Neonatal Admission in the Tamale Teaching Hospital. Clinics Mother Child Health 13: 252. doi:10.4172/2090-7214.1000252

16. Susana DP and Luiz F. Risk factors for neonatal death in neonatal intensive care unit according to survival analysis.Rev Bras Ter Intensiva,2010: 22(1) 19-26.

17. Sabzehei MK, Basiri B, Shoukohi M, Torabian S, Razavi Z. Factors affecting the complications of interhospital transfer of neonates referred to the Neonatal Intensive Care Unit of Besat Hospital in 2012-2013. J Clin Neonatol 2016;5:238-42.

18. Ike $E$ and Mudupe O. Pattern of disease and care outcomes of neonates admitted in the special care baby unit of University college hospital, Ibadan,Nigeria from 2007-2011. IOSR Journal of nursing and health science vol,4, issue 3, 2015.

\section{Tables}

[Please see the supplementary files section to view the tables.] 
Figures

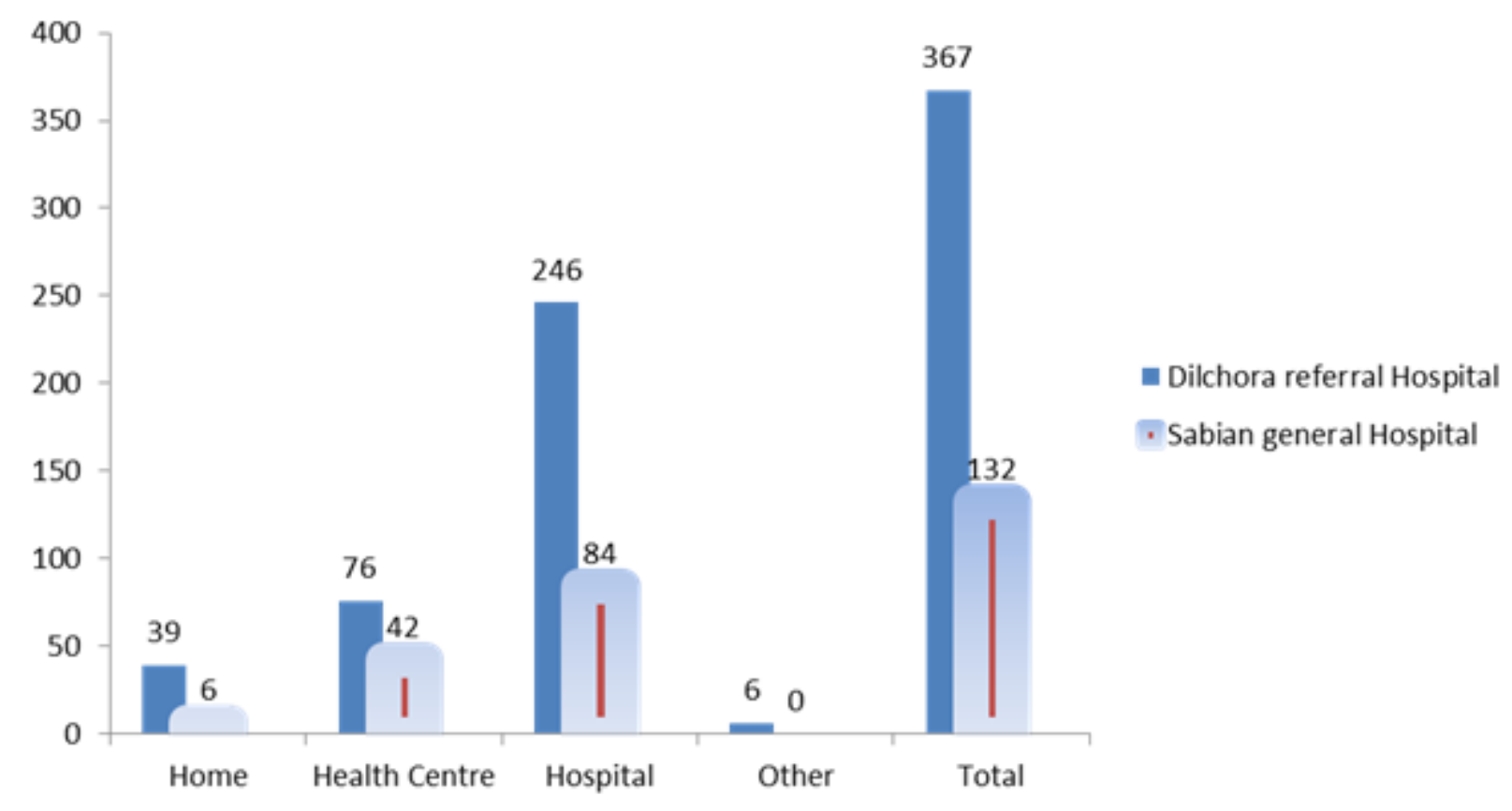

Figure 1

Place of Delivery for Neonates admitted in Dire Dawa public Hospitals from January 2017 to January 2019. 


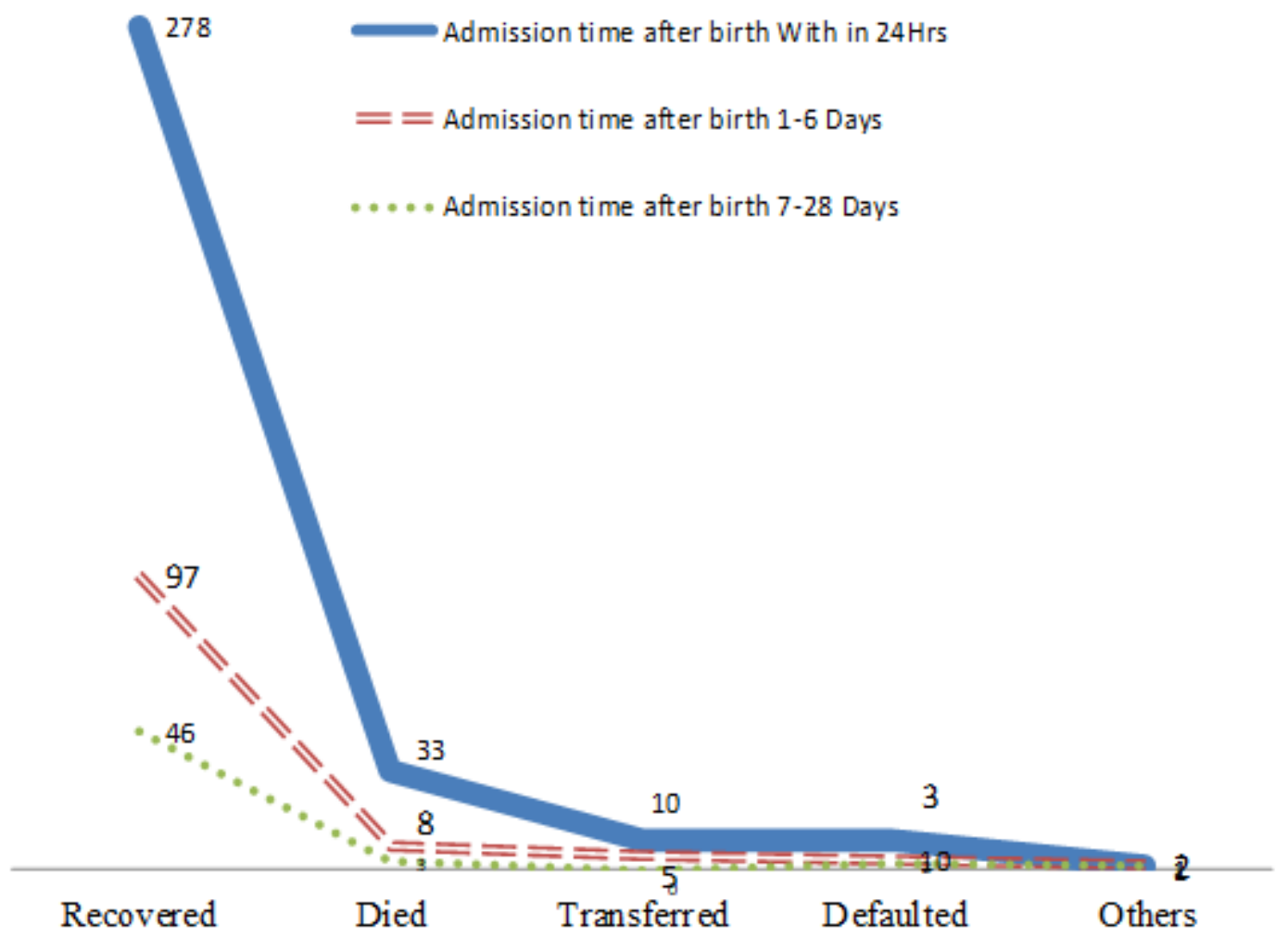

\section{Figure 3}

Admission time after birth and treatment outcome of neonates that were admitted in neonatal intensive care of Dire Dawa Public hospitals from January 2017 to January 2019. 


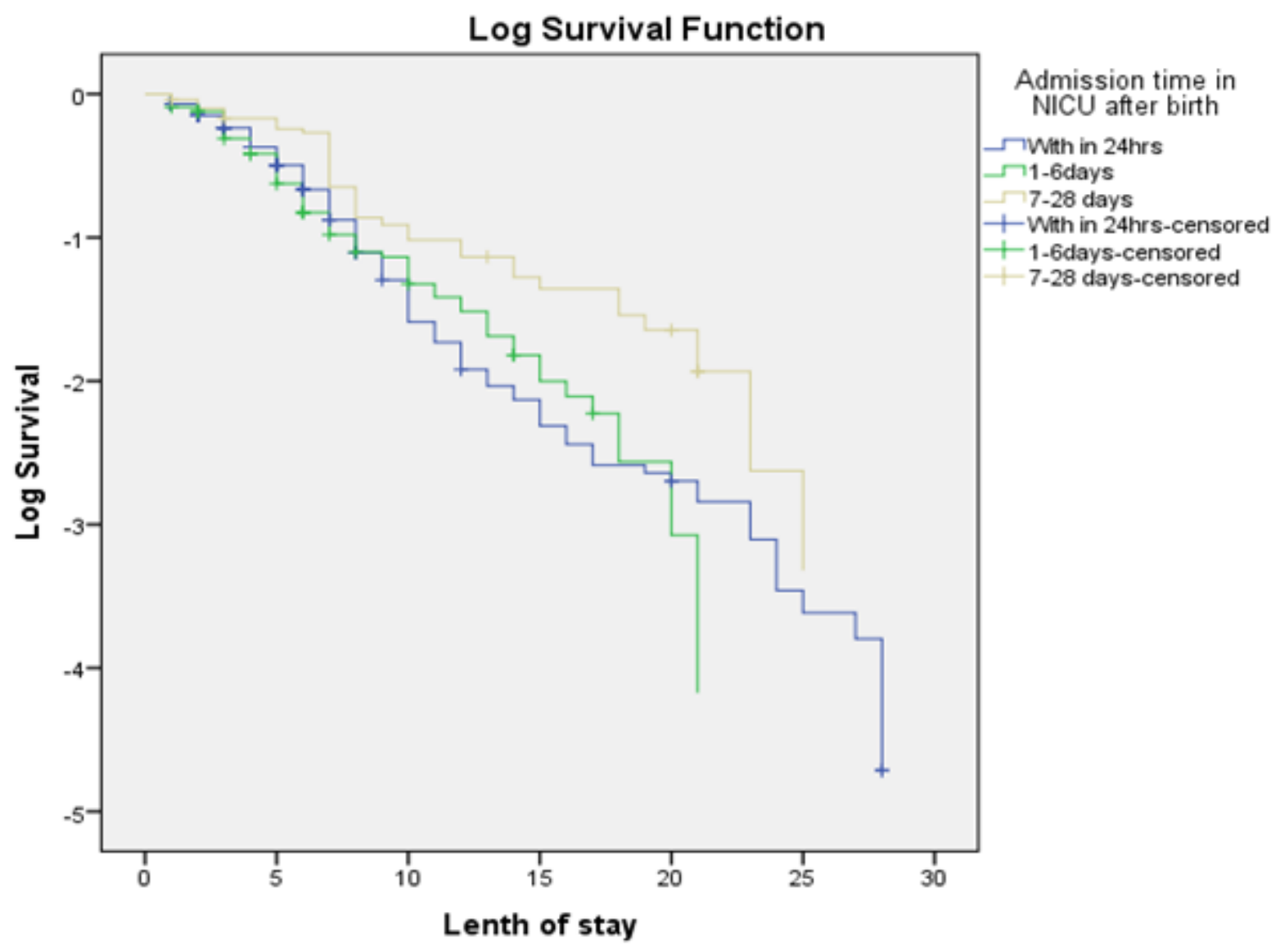

Figure 5

Hazard function estimates graph for neonates admitted to neonatal intensive care units with admission time after births in Dire Dawa Public hospitals, from January 2017 to January 2019.

\section{Supplementary Files}

This is a list of supplementary files associated with this preprint. Click to download.

- Tables.docx

- Tables.docx 